\title{
THERMO GRAVIMETRY-DIFFERENTIAL SCANNING COLORIMETRY-MASS SPECTROSCOPY-A REVIEW
}

\author{
K. BHAVYA SRI ${ }^{*}$, G. SRI VANI SHAILAJA ${ }^{2}$ \\ *1,2Department of Pharmaceutical Analysis, RBVRR Women's College of Pharmacy, Barkatpura, Hyderabad 500027 \\ Email: bhavya.khagga@gmail.com
}

Received: 05 May 2021, Revised and Accepted: 03 Jul 2021

\begin{abstract}
Thermogravimetric analysis is an analytical technique used to determine a material's thermal stability and its fraction of volatile components by monitoring the weight change that occurs as a sample is heated at a constant rate. Differential scanning colorimetry analysis is used to measure melting temperature, the heat of fusion, latent heat of melting, reaction energy etc. Mass spectroscopy is a powerful analytical tool with many applications in pharmaceuticals and biomedical fields. The increase in sensitivity and resolution of the instrument as opened new dimensions in analysis of pharmaceuticals and complex metabolites of biological systems. Thermo gravimetry coupled with differential scanning colorimetry and Quadra pole mass spectrometry was applied to monitor the thermal stability and chemical properties of natural polymers isolated from chemically different soils. The TGA/DSC, when coupled with MS generic multiple ions from the sample under investigation, it then separates them according to a specific mass-to-charge ratio. The coupled instrument is used for simultaneous identification of organic compounds, used to evaluate the physical properties, degradation stability of powder coating.
\end{abstract}

Keywords: Thermogravimetry, Differential scanning colorimetry, Quadra pole mass spectroscopy, DSC sensor, Thermal stability, Degradation stability

(C) 2021 The Authors. Published by Innovare Academic Sciences Pvt Ltd. This is an open-access article under the CC BY license (https://creativecommons.org/licenses/by/4.0/)

DOI: https://dx.doi.org/10.22159/ijcpr.2021v13i5.1883 Journal homepage: https://innovareacademics.in/journals/index.php/ijcpr

\section{INTRODUCTION}

The thermogravimetric analyzer is an essential laboratory tool used for material characterization. TGA is used as a technique to characterize materials used in various environmental, food, pharmaceutical and petrochemical applications. PerkinElmer is the leader of TGA. Thermogravimetric analysis or thermal gravimetric analysis is a method of thermal analysis in which the mass of the sample is measured over time as the temperature changes. This measurement provides information about physical phenomena, such as phase transitions, absorption, adsorption and desorption; as well as chemical phenomena including chemisorption, thermal decomposition and solid-gas reaction [1].

The differential scanning colorimeter is a fundamental tool in thermal analysis. It is used in many industries-from pharmaceuticals and polymers to nanomaterials and food products. The information these instruments generate is used to understand amorphous and crystalline behavior, polymorph and eutectic transitions, curing and degree of cure, and many other material properties used to design, manufacture and test products. DSC analysis is used to measure melting temperature, the heat of fusion, latent heat of melting, reaction energy and temperature, glass transition temperature etc.

The foundation of mass spectroscopy was laid in 1898. The study of gas discharges in the mid19 th century led to the discovery of anode and cathode rays, which turned out to be, positive ions and electrons. Improved capabilities in the separation of these positive ions enabled the discovery of stable isotopes of the elements. The first such discovery was with the element neon which was shown by mass spectrometry to have at least two stable isotopes. Mass spectrometers were used in the Manhattan project for the separation of isotopes of Uranium necessary to create the atomic bond [2].

\section{Scope}

\section{Thermogravimetric analysis}

Thermogravimetric analysis or thermal gravimetric analysis is a method of thermal analysis (is a branch of materials science where the properties of materials are studied as they change the temperature) in which the mass of the sample is measured over time as temperature changes. TGA is a method of thermal analysis in which changes in physical and chemical properties of materials are measured as a function of increasing temperature or as a function of time. Thermogravimetric analysis is performed by gradually raising the temperature of a sample in the furnace as its weight is measured on an analytical balance that remains outside of the furnace. In TGA, mass loss is observed if a thermal event involves the loss of a volatile component. TGA is an analytical technique used to determine a materials thermal stability and its fraction of volatile components by monitoring the weight changes that occurs as a sample is heated at a constant rate. The below (fig. 1) explains the instrumentation of TGA.

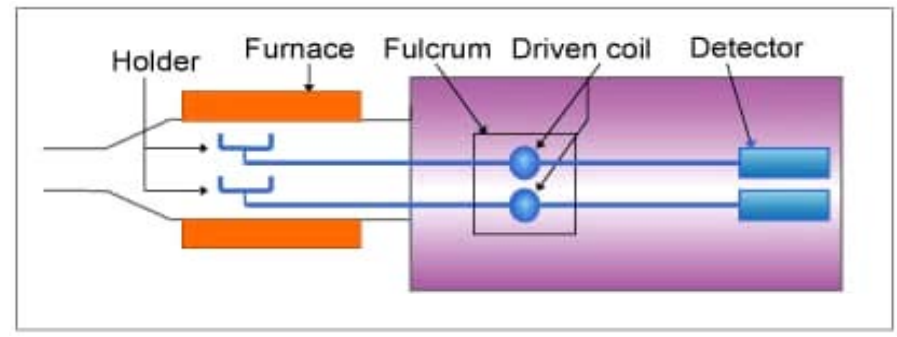

Fig. 1: Thermo gravimetric analysis 


\section{TGA basics}

Measures changes in weight in relation to changes in temperature. The measured weight loss gives information on changes in sample composition, thermal stability, kinetic parameters for chemical reactions in the sample.

\section{Mechanisms of weight change in TGA}

\section{a. Weight loss}

Decomposition: The breaking apart of chemical bonds.

Evaporation: The loss of volatiles with elevated temperature.

Reduction: Interaction of sample to reducing atmosphere (hydrogen, ammonia, etc.)

Desorption.

\section{b. Weight gain}

Oxidation: Interaction of the sample with an oxidizing atmosphere.

Absorption or adsorption.

\section{Differential scanning colorimetry}

Differential scanning calorimetry is a thermoanalytical technique in which the difference in the amount of heat required to increase the temperature of a sample and reference is measured as a function of temperature. Both the sample and reference are maintained at nearly the same temperature throughout the experiment. DSC measures the amount of heat required to raise the temperature of a sample; then, it determines the amount of heat required to raise the temperature of reference material. This allows the detection of transitions such as melts, glass transitions, phase changes and curing. DSC is used to measure enthalpy changes due to changes in physical and chemical properties of a material as a function of temperature or time. The method allows you to identify and characterize materials. DSC is fast, very sensitive and easy to use. The (fig. 2) explains the instrumentation of DSC.

DSC heat flow: $\mathrm{dH} / \mathrm{dt}=\mathrm{Cp} \mathrm{dT} / \mathrm{dt}$

$\mathrm{dT} / \mathrm{dt}=$ Heating rate; $\mathrm{dH} / \mathrm{dt}=\mathrm{DSC}$ heat flow signal; $\mathrm{Cp}=$ sample heat capacity.

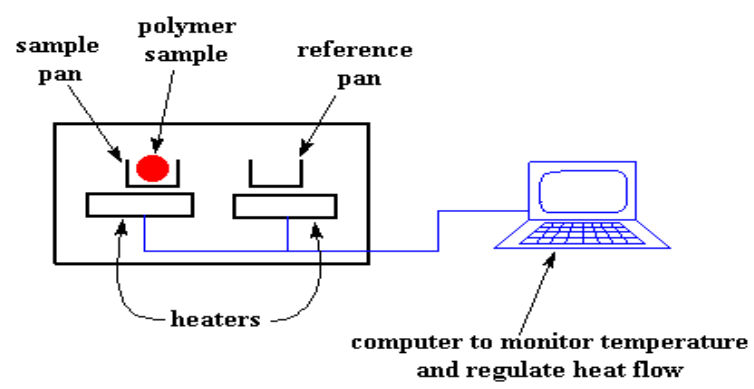

Fig. 2: Differential scanning colorimetry

\section{Mass spectrometry}

MS is an analytical technique that measures the mass-to-charge ratio of ions. It generates multiple ions from the sample under investigation, it then separates them according to their specific mass-to-charge ratio $(\mathrm{m} / \mathrm{z})$, and then records the relative abundance of each ion type. A beam of electrons will be bombarded in the analyte compound and it will lead to the removal of one electron from the analyte. Due to the removal of the electron, the molecule will be positively charged and known as a molecular ion. Molecular ions will be fragmented. The (fig. 3) explains the instrumentation of MS.

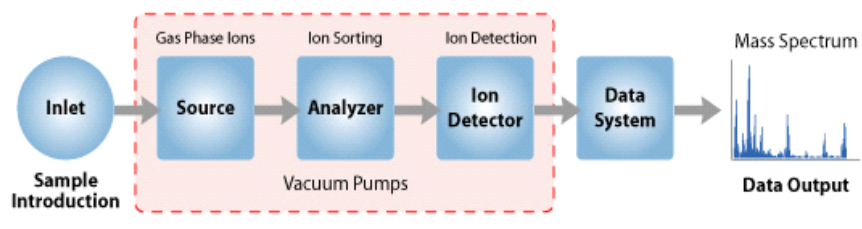

Fig. 3: Mass spectrometry

\section{Principle of TGA-DSC-MS}

TGA measures mass of a sample as it is heated, cooled or held at a constant temperature in defined atmosphere. A TGA instrument consists of furnace and a highly sensitive balance. When TGA coupled with DSC instrument, the scale pan is replaced by a DSC sensory. The DSC sensor measures the temperature difference between the sample and the reference. This signal is calibrated and adjusted to a heat flow signal. The MS generates multiple ions from the sample under investigation, it then separates them according to their specific mass-to-charge ratio $(\mathrm{m} / \mathrm{z})$, and then records the relative abundance of each ion type.

\section{TGA-DSC technique}

Simultaneous TGA-DSC measures both heat flow and weight changes in a material as a function of temperature or time in a controlled atmosphere. Simultaneous measurement of these two material properties not only improves productivity but also simplifies interpretation of the results. The complimentary information obtained allows differentiation between endothermic and exothermic events which have no associated weight loss (e. g: melting and crystallization) and those which involve a weight loss (e. g: degradation) [3].

\section{Instrumentation}

\section{Sample and reference pan}

Crucibles are made of quartz, aluminum, alumina, platinum with 80 , 90 or 100 micro liters are available with $5 \mathrm{~mm}$ diameter and $8 \mathrm{~mm}$ height for small crucibles. Large crucibles with 1000 microliters, 8 $\mathrm{mm}$ diameter and $27 \mathrm{~mm}$ height are available.

\section{Photodiode}

A photodiode is a semiconductor device that converts light into an electrical current. When a photon of ample energy strikes the diode, it makes a couple of electron-hole. Therefore, holes in the region move towards the anode, and electron move towards the cathode, and a photocurrent will be generated. 
Photodiode are similar to regular semiconductor diodes except that they may be either exposed or packed with a window or optical fiber connection to allow light to reach the sensitive part of device. Many diodes designed for use specially as a photodiode use a PN junction rather than a p-n junction, to increase the speed of response. A photodiode is designed to operate in reverse bias [4].

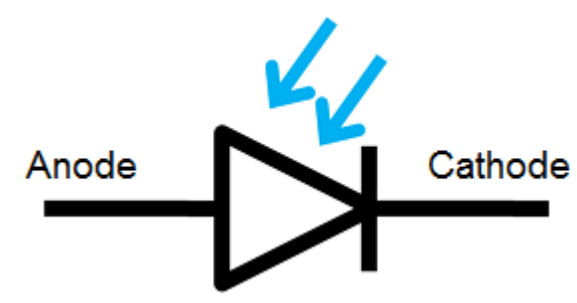

Fig. 4: Photodiode symbol

\section{Sensor}

Sensors are sophisticated devices that are frequently used to detect and respond to electrical or optical signals. A sensor converts the physical parameters (temperature, humidity, speed, etc.) into a signal which can be measured electrically [5].

\section{Purging}

Purging is the act of removing the contents of a pipe or container and replacing it with another gas or liquid. Purging is crucial in pipeline, piping, welding and industrial process. It removes contaminants from the piping and vessels, which reduces chances of corrosion. Purging can prevent a hazardous mixture of gas and air. The purge gas is inert. The most common purge gases commercially available in large quantities are nitrogen and carbon dioxide. Other inert gases, eg: argon or helium may be used [6].

\section{Furnace}

A furnace is a device used to heat and melt metal ore to remove gauge (commercially valueless material in which ore is found). The heat energy to fuel a furnace may be supplied directly by fuel combustion, by electricity such as the electric arc furnace, or through induction heating in induction furnaces. An induction furnance is an electrical furnace in which the heat is applied by induction heating of metal [7-9]. Induction furnace capacity range from less than one kg to a hundred tonnes, and are used to melt iron and steel, copper, aluminium, and precious metals.

\section{Quadrupole}

The quadrupole mass spectrometer is the most common mass analyzer, as shown in (fig. 5). Its compact size, fast scan rate, high transmission efficiency, and modest vacuum requirements are ideal for small inexpensive instruments. Most quadrupole instruments are limited to unit $\mathrm{m} / \mathrm{z}$ resolution and have mass range of $\mathrm{m} / \mathrm{z} 1000$. Many benchtop instruments have a mass range of $\mathrm{m} / \mathrm{z} 500$ but research instruments are available with mass range up to $\mathrm{m} / \mathrm{z} 4000$ [10].

In MS, an electric field accelerates ions out of the source region and into the quadrupole analyzer. The analyzer consists of four rods or electrodes arranged across from each other. As the ions travel through the quadrupole, they are filtered according to their $\mathrm{m} / \mathrm{z}$ value so that only a single $\mathrm{m} / \mathrm{z}$ value ion can strike the detector. The $\mathrm{m} / \mathrm{z}$ value transmitted by the quadrupole is determined by the radio frequency (RF) and direct current (DC) voltages applied to the electrodes. These voltages produce an oscillating electric field that functions as a band pass filter to transmit the selected $\mathrm{m} / \mathrm{z}$ value [11].

The $\mathrm{RF}$ voltage rejects or transmits ions according to their $\mathrm{m} / \mathrm{z}$ value by alternately focusing them in different planes. The four electrodes are connected in pairs and the RF potential is applied between these two pairs of electrodes. During the first part of RF cycle the top and bottom rods are at a positive potential and the left and right rods are at a negative potential. This squeezes positive ions into the horizontal plane. During the second half of the RF cycle, the polarity of the rods is reversed. This changes the electric field and focuses the ions in the vertical plane. The quadrupole field continues to alternate as the ions travel through the mass analyzer. This causes the ions to undergo a complex set of motions that produces a three-dimensional wave.

The quadrupole field transmits selected ions because the amplitude of this three-dimensional wave depends upon the $\mathrm{m} / \mathrm{z}$ value of the ion, the potentials applied, and the RF frequency. By selecting an appropriate RF frequency and potential, the quadrupole acts like a high pass filter, transmitting high $\mathrm{m} / \mathrm{z}$ ions and rejecting low $\mathrm{m} / \mathrm{z}$ ions. The low $\mathrm{m} / \mathrm{z}$ ions have a greater acceleration rate so the wave for these ions has a greater amplitude. If the amplitude is great enough the ions will collide with the electrodes and cannot reach the detector. The low $\mathrm{m} / \mathrm{z}$ value cutoff the quadrupole is changed by adjusting the RF potential or the RF frequency. Any ions with a m/z greater than this cutoff are transmitted by the quadrupole.

A DC voltage is also applied across the rods of the analyzer. This potential, combined with the RF potential, acts like a low pass filter to reject high $\mathrm{m} / \mathrm{z}$ ions. Because they respond quickly to the changing RF field the motion of the low $\mathrm{m} / \mathrm{z}$ ions is dominated by the RF potential. This motion stabilizers their trajectory by refocusing each time the RF potential changes polarity. Because low $\mathrm{m} / \mathrm{z}$ ions are quickly refocused, the DC potential does not affect these ions. High $\mathrm{m} / \mathrm{z}$ ions, however, do not refocus as quickly during the $\mathrm{RF}$ cycle. The DC potential has a greater influence on their trajectory and they slowly drift away from the center of the quadrupole. At the end of the analyzer, they are too far off-axis to strike the detector.

The combination of high and low pass filters produced by the RF and DC potential is adjusted to only transmit the selected $\mathrm{m} / \mathrm{z}$ value. All ions above or below the set $\mathrm{m} / \mathrm{z}$ value are rejected by the quadrupole filter. The RF and DC field are scanned to collect a complete mass spectrum. Quadrupole mass analyzers are often called mass filters because of the similarity between $\mathrm{m} / \mathrm{z}$ selection by a quadrupole and wavelength selection by an optical filter or frequency selection by an electronic filter.

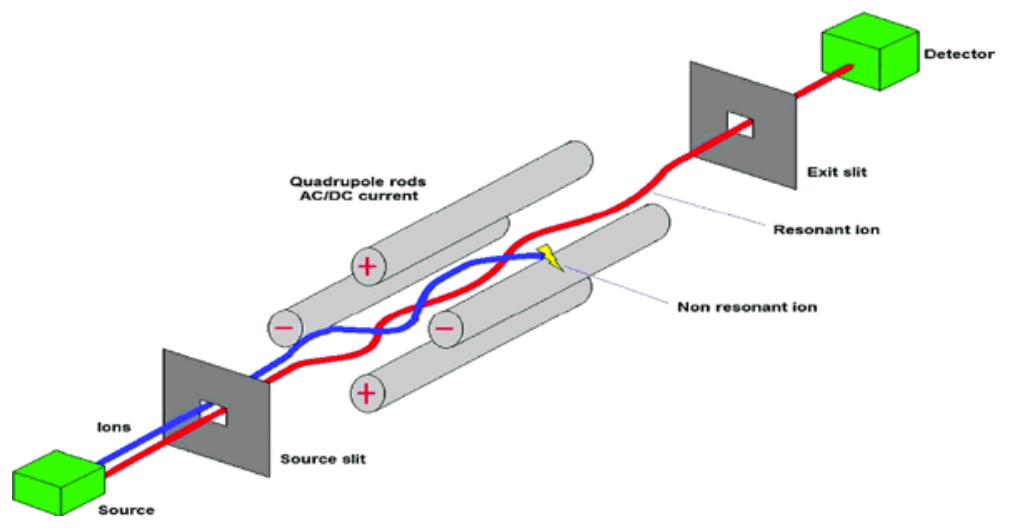

Fig. 5: Quadrupole mass analyzer 


\section{Detector}

\section{Electron multiplier [12]}

A electron multiplier is used to detect the presence of ion signals emerging from the mass analyzer of a mass spectrometer. It is essentially the "eyes" of the instrument. The task of electron multiplier is to detect every ion of the selected mass passed by the mass filter. How efficiently the electron multiplier carries out this task represents a potentially limited factor on the overall system sensitivity. Consequently, the performance of the electron multiplier can have a major influence on the overall performance of the mass spectrometer. The basic physical process that allows an electron multiplier to operate is called secondary electron emission. When a charged particle strikes a surface it causes secondary electrons to be released from atoms in the surface layer. The number of secondary electrons released depends on the type of incident primary particle, its energy and characteristic of the incident surface.

Dynodes made up of copper-beryllium, which transduces the initial ion current and electron emitted by first dynode is focused magnetically from dynode to the next. Final cascade current amplified more than million times.

There are two basic forms of electron multiplier that are commonly used in mass spectrometer. They are discrete-dynode electron multiplier and continuous-dynode electron multiplier. All ETP electron multiplier are of discrete-dynode type. A typical discretedynode electron multiplier has between 12 and 24 dynodes and is used with an operating gain of between 104 and 108, depending on the application.

As this is most sensitive detector. It protected from stray ions, neutral and cosmic rays. Then the background count rate is normally less than the 0.01 counts per second. However multiplier must also be protected from intense ion beams as these can rapidly leads to its destruction.

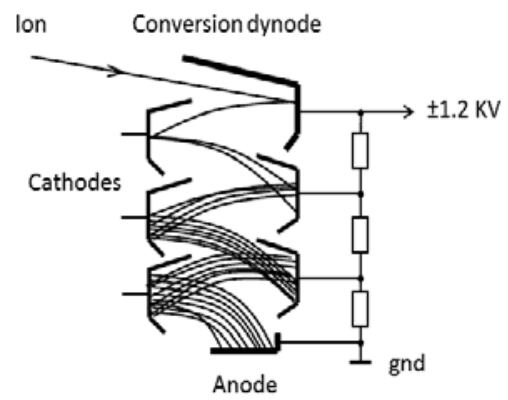

Fig. 6: Electron multiplier

\section{Applications}

a. TG-DSC-MS were used to characterize the hydrogen adsorption temperature and capacity of Ticr 1.2 alloy.

b. They are useful to evaluate physical properties, curing behaviour and degradation stability of powder coating.

c. The thermal behaviour of anticancer drugs was measured by thermo gravimetric, differential scanning spectrometry to explore the application of TG-DTA in nano medicine. d. The TGA-DSC-MS is only able to detect an extremely small amount of substances. This technique is ideal for the characterization of all types of volatile compounds.

\section{CONCLUSION}

a. Thermogravimetry coupled with differential scanning colorimetry and Quadra pole mass spectrometry was applied to monitor the thermal stability and chemical properties of natural polymers, isolated from chemically different soils.

b. The TGA/DSC sensor measures the temperature difference between the sample and reference.

c. The TGA/DSC, when coupled with MS generates multiple ions from the sample under investigation, it then separates them according to their specific mass-to-charge ratio.

d. The coupled instrument is used for simultaneous identification of organic compounds, used to evaluate the physical properties, degradation stability of powder coating.

\section{ACKNOWLEDGEMENT}

I want to acknowledge our beloved principal Prof. M. Sumakanth RBVRR women's college of pharmacy for giving me the opportunity for writing this review paper.

\section{FUNDING}

Nil

\section{AUTHORS CONTRIBUTIONS}

All the authors have contributed equally.

\section{CONFLICT OF INTERESTS}

\section{Declared none}

\section{REFERENCES}

1. Coast AW, Redfern JP. Thermogravimetric analysis: a review. Analyst 1963;88:906-24.

2. Hewlett and Anderson; 1962. p. 10-2.

3. Gill PS, Marcozzi CL. Simultaneous measurement of weight loss and delta temperature. J Thermal Anal 1993;40:923-9.

4. James F. Fundamentals of linear electronics: integrated and discrete. Cengage Learning; 2001.p. 91.

5. IF Akyildiz, W Su. A power aware enchanced routing (PAER) protocol for sensor network, Georgia Tech Technical; 2002.

6. NFPA 56. Standard for fire and explosion prevention during cleaning and purging of flammable gas piping system. National Fire Protection Association; 2020.

7. Laughton MA, Warne DF. Electrical engineer's reference books. $16^{\text {th }}$ Ed. Newnes; 2002. p. 17-9.

8. Campbell, Flake C. Metals fabrication: understanding the basics. ASM International; 2013. p. 63-5.

9. Bauccio, Michael. ASM metals reference book. $3^{\text {rd }}$ Ed. Amercian society for metals; 1993. p. 50.

10. Steel C, Henchman MJ. Design and operation of a portable quadrupole mass spectrometer for the undergraduate curriculum. Chem Educ 1998;75:1049-54.

11. De Hoffmann, Edmond, Vincent Stroobant. Mass spectrometry: principles and applications. $2^{\text {nd }}$ ed. Toronto: John Wiley and Son's Ltd; 2003. p. 65.

12. Jyothika Rajawat, Gagandeep Jhingan. Data processing handbook for complex biological data sources; 2019. 